\title{
Terapia com agentes biológicos na criança e no adolescente
}

\author{
Treatment with biologic agents in child and adolescent
}

Ricardo Maisse Suehiro ${ }^{1}$, Nádia Emi Aikawa², Jozélio Freire de Carvalho ${ }^{3}$, Clovis Artur A. da Silva ${ }^{4}$

\section{RESUMO}

Objetivo: Revisar os mecanismos fisiopatológicos e novos alvos terapêuticos, os agentes biológicos disponíveis, principais indicações e a evidência científica atual para o uso de terapias biológicas na população pediátrica.

Fontes de dados: Pesquisa na base de dados Medline e SciELO, nas línguas inglesa e portuguesa, entre 2000 e 2009. As palavras-chave usadas foram "agentes biológicos", "crianças" e "adolescentes".

Síntese dos dados: Os agentes biológicos são uma importante opção terapêutica para tratar as doenças autoimunes refratárias às terapias convencionais na infância e na adolescência. Com exceção da artrite idiopática juvenil, a maioria dos estudos em outras doenças autoimunes não é controlada.

Conclusões: Os agentes biológicos têm demonstrado eficácia no tratamento de doenças autoimunes pediátricas como artrite idiopática juvenil, miopatias idiopáticas inflamatórias, lúpus eritematoso juvenil, vasculites, uveítes crônicas, doenças inflamatórias intestinais e púrpura trombocitopênica imune crônica, assim como no linfoma não-Hodgkin. Considerando-se o custo elevado e os potenciais eventos adversos, o uso desses agentes deve ser individualizado e acompanhado por especialista.

Palavras-chave: terapia biológica; doenças autoimunes; criança; adolescente.

\section{ABSTRACT}

Objective: To review the physiopathology and new therapeutical targets, the available biologic agents, the main indications and the current scientific evidence for the use of biological therapies in the pediatric population.

Data sources: A bibliographical search was obtained from Medline and SciELO databases in English and Portuguese from 2000 to 2009. The key-words included were "biologic agent", "children" and "adolescent".

Data synthesis: Biologic agents are important therapeutic options to treat refractory autoimmune diseases to conventional therapies in childhood and adolescence. Excluding juvenile idiopathic arthritis, the majority of studies in other autoimmune diseases are uncontrolled trials.

Conclusions: Biologic agents have shown efficacy in the treatment of pediatric autoimmune diseases such as juvenile idiopathic arthritis, idiopathic inflammatory myositis, juvenile systemic lupus erythematosus, vasculitis, chronic uveitis, inflammatory bowel diseases, and chronic immune thrombocytopenic purpura, as well as in non-Hodgkin lymphoma. Considering the high cost and the potential adverse events, the choice to use them must be individualized and followed by a specialist.

Key-words: biological therapy; autoimmune diseases; child; adolescent.
Instituição: Disciplina de Reumatologia do Departamento de Pediatria da Faculdade de Medicina da Universidade de São Paulo (USP), São Paulo, SP, Brasil

'Doutor; Médico Reumatologista Pediátrico do Departamento de Pediatria da Faculdade de Medicina da USP, São Paulo, SP, Brasil

${ }^{2}$ Mestranda; Médica Reumatologista Pediátrica do Departamento de Pediatria da Faculdade de Medicina da USP, São Paulo, SP, Brasil

${ }^{3}$ Doutor; Professor colaborador da Disciplina de Reumatologia da FMUSP, São Paulo, SP, Brasil

${ }^{4}$ Livre-Docente; Professor do Departamento de Pediatria da Faculdade de Medicina da USP; Médico Responsável pela Unidade de Reumatologia Pediátrica do Instituto da Criança do Hospital das Clínicas da Faculdade de Medicina da USP, São Paulo, SP, Brasil
Endereço para correspondência:

Clovis Artur A. da Silva

Avenida Dr. Enéas Carvalho de Aguiar, 647 - Cerqueira César

CEP 05403-900 - São Paulo

E-mail: clovis.silva@icr.usp.br

Conflitos de interesse: Ricardo Maisse Suehiro é Gerente Médico-Científico de Imunologia da Bristol-Myers Squibb Farmacêutica. Nádia Emi Aikawa, Jozélio Freire de Carvalho e Clovis Artur Almeida da Silva declaram não haver nenhum conflito de interesse.

Recebido em: 10/12/08

Aprovado em: 25/4/09 


\section{Introdução}

Uma nova era no tratamento de doenças inflamatórias imunomediadas inciou-se com a introdução dos agentes biológicos. $\mathrm{Na}$ faixa etária pediátrica, esses medicamentos têm sido utilizados particularmente em doenças que não são controladas após uso combinado de drogas ou em monoterapia de anti-inflamatórios não-hormonais, corticosteroides, drogas modificadoras do curso da doença, imunossupressores e/ou gamaglobulina intravenosa.

Dentre as doenças que podem se beneficiar dos agentes biológicos, destacam-se: artrite idiopática juvenil (AIJ), miopatias inflamatórias idiopáticas (MII), lúpus eritematoso sistêmico (LES) e juvenil (LESJ), vasculites (como doença de Kawasaki e arterite de Takayasu), uveítes crônicas, doenças inflamatórias intestinais (DII), linfoma não-Hodgkin (LNH) e púrpura trombocitopênica imune (PTI) crônica. Até o presente momento, estudos randomizados, duplo-cegos controlados por placebo com agentes biológicos em crianças e adolescentes foram realizados apenas em AIJ.

\section{Mecanismos fisiopatológicos e alvos terapêuticos}

A melhor compreensão da fisiopatologia de doenças autoimunes nos adultos, como a artrite reumatoide (AR) e o LES, tem permitido a descoberta de novos potenciais alvos terapêuticos. Nessas doenças, são importantes, principalmente, células $T$, células $B$, citocinas e quimiocinas.

$\mathrm{Na} \mathrm{AR}$, a apresentação de um antígeno às células $\mathrm{CD} 4+$ é o evento inicial que determina ativação da célula T. Além do primeiro sinal [apresentação do antígeno ligado ao complexo de histocompatibilidade principal (do inglês major histocompatibility complex, MHC) ao receptor da célula T], um segundo sinal é necessário para a ativação da célula T. Esse segundo sinal é a interação de moléculas coestimulatórias, como o CD80 e o CD86, na célula apresentadora de antígeno, e o CD28, na célula T. O abatacepte (CTLA4-Ig) é o primeiro de uma nova classe de biológicos, a dos moduladores seletivos da coestimulação, ligando-se ao CD80/CD86 na superfície da célula apresentadora de antígeno e, consequentemente, impedindo a ativação da célula T. Outros inibidores de moléculas coestimulatórias em investigação são o RhuDex e o alefacepte (esse último, indicado para psoríase de adultos, liga-se ao receptor $\mathrm{CD} 2$ nas células $\mathrm{T})^{(1-5)}$.

Há ainda moléculas de adesão que fornecem sinais de transdução e amplificação necessários à completa ativa- ção da célula T. Essas moléculas incluem, por exemplo, o LFA-1 (CD11a/CD18) na célula T e a molécula de adesão 1 (ICAM-1) na célula apresentadora de antígeno, assim como o CD2 na célula T e o LFA-3 na célula apresentadora de antígeno. Tais moléculas têm sido exploradas como alvo terapêutico, constituindo os moduladores de móleculas de



As células $T$ ativadas na sinóvia de pacientes com AR secretam citocinas e estimulam outras células que se tornam ativadas. Além dos agentes anti-TNF disponíveis atualmente (etanercepte, infliximabe e adalimumabe), novos medicamentos anti-TNF (golimumabe, certolizumabe, tgAAC94) e novas drogas contra citocinas (como IL-6, IL-10, IL-12, IL-10, IL-23, IL-17, interferon - IFN- $\gamma$ e IL-18) têm sido estudados e consistem nos modulatores de citocinas (exemplos: tocilizumabe, rilonacepte, AMG108, ACZ885, AMG-714, AIN457, STA-5326, ABT-874, IL-18BP, fontolizumabe e $\mathrm{K}-832)^{(1-5)}$.

Por sua vez, as citocinas ligam-se a um receptor específico na superfície da célula, resultando em resposta celular com produção e secreção de outra citocina. Essa ligação é seguida por uma série de fenômenos por meio de sistemas de transdução que transferem a informação da superfície celular ao núcleo e que estão organizados como complexos de proteínas kinases sequenciais. Dessas proteínas, as mais importantes são as MAP kinases (MAPK). Um grande número de compostos em desenvolvimento (CP-690550, R788) bloqueia a via de sinalização das MAPK, constituindo os inibidores da p38 MAPK (VX-702, SCIO-469, R1503, GSK/681323) ${ }^{(1,2)}$.

As células B são também importantes na fisiopatologia da AR e do LES. Além de produzirem imunoglobulinas, elas também podem agir como células apresentadoras de antígenos e secretar citocinas, perpetuando a inflamação. A identificação de marcadores específicos para as células B tem permitido a remoção seletiva de uma população específica dessas células. Uma proteína integrante de membrana, a CD20, é responsável pela ativação e diferenciação de células B. A proteína estimuladora de linfócitos B (BLyS), também conhecida como fator ativador de células B (BAFF), é um membro da família do TNF. Juntamente ao receptor transmembrane activator and calcium modulator and cyclophilin ligand interactor (TACI) forma o complexo ligante-receptor, com múltiplas funções. O BLyS, essencial para a ativação e diferenciação das células $\mathrm{B}$, age como um sinal coestimulatório nas respostas mediadas pelas células T. Vários compostos estão em desenvolvimento (ocrelizumabe, ofatumumabe, 
belimumabe, BR3-Fc, atacicepte e TRU-015) que, assim como o rituximabe (anti-CD20), bloqueiam as células B e compõem a classe de depletores das células $\mathrm{B}$, podendo ser utilizados no LES, LESJ, AR e PTI crônica ${ }^{(1-5)}$.

A formação de imunocomplexos na sinóvia determina ativação do complemento e libera fatores quimiotáxicos, como C5a, responsáveis pelo influxo de células inflamatórias na sinóvia. Os moduladores do complemento são pequenas moléculas que têm como alvo fatores do complemento (como C5a, cujo medicamento é o eculizumabe). Essas drogas estão em desenvolvimento para tratamento de doenças como AR e $\operatorname{LES}^{(1,3-5)}$.

$\mathrm{Na}$ sinóvia de pacientes com AR, citocinas e quimiocinas são secretadas e atingem condrócitos e osteoclastos, liberando colagenases e metaloproteinases para a degradação da cartilagem e do osso. Drogas que inibem as quimiocinas (MLN1202, MLN3897 e AVE1701) e as metaloproteinases compõem uma nova classe em desenvolvimento ${ }^{(1)}$.

O TNF $\alpha$ é uma citocina importante que estimula os osteoclastos por ativação da via RANK/RANKL. Agentes que inibem o RANK (como denosumabe) constituem um novo grupo terapêutico, o de moduladores do RANK/RANKL, que têm sido estudados em adultos com osteoporose ${ }^{(1,3)}$.

$\mathrm{E}$, finalmente, uma última classe de novas drogas inclui pequenas moléculas que inibem a transcrição de fatores como NF-KB (CF101) ou medicamentos que induzem tolerância (AT-001 ou DNA JP1), utilizados na AR e no LES $^{(1,3,5)}$.

Os mecanismos fisiopatológicos da AR e LES, potenciais alvos terapêuticos e agentes biológicos disponíveis e em estudo estão sumarizados na Tabela 1.

\section{Agentes biológicos disponíveis e indicações}

Atualmente, existem três classes de agentes biológicos disponíveis no Brasil: bloqueadores de TNF $\alpha$ ou drogas anti-TNF (etanercepte, infliximabe e adalimumabe), depletores de células B (rituximabe) e moduladores seletivos da coestimulação (abatacepte) $)^{(6-9)}$.

Nos Estados Unidos, etanercepte, adalimumabe e abatacepte foram aprovados pelo Food and Drug Administration (FDA) para AIJ de qualquer forma de início e com evolução poliarticular ativa (envolvimento de cinco ou mais articulações e com artrite persistente). Porém, no Brasil, até o presente momento, o etanercepte é o único agente biológico aprovado pela Agência Nacional de Vigilância Sanitária (Anvisa) para uso em crianças e adolescentes com $\mathrm{AIJ}^{(8,9)}$.

\section{Artrite Idiopática Juvenil}

A AIJ é a principal causa de artrite crônica na faixa etária pediátrica, com alto impacto físico, mental, emocional, econômico e social nos pacientes e familiares. Os agentes biológicos são indicados para os casos não-responsivos aos anti-inflamatórios não-hormonais e/ou drogas antirreumáticas modificadoras do curso da doença (DMARDs) ou imunossupressores, como metotrexato (MTX), sulfassalazina, ciclosporina, leflunomide etc. ${ }^{(9)}$. A tabela inclui os agentes biológicos disponíveis para tratamento na AIJ e suas principais características.

Para avaliar a resposta ao tratamento na AIJ, utiliza-se o critério pediátrico validado pelo American College of Rheumatology (ACR), conhecido como ACR Pediatric, no qual seis parâmetros são avaliados: 1) número de articulações ativas; 2) número de articulações com limitação de movimento; 3) avaliação global do médico; 4) avaliação global do paciente ou dos pais; 5) Children's Health Assessment Questionnaire (CHAQ); e 6) provas de atividade inflamatória [velocidade de hemossedimentação (VHS) ou proteína $\mathrm{C}$ reativa (PCR)]. Para preencher os critérios ACR Pediatric 30, 50, 70 ou 90, deve haver melhora de 30, 50, 70 ou $90 \%$, respectivamente, em pelo menos três dos seis parâmetros. Além disso, para preencher esses critérios, pode haver piora de apenas um destes parâmetros em $>30 \%{ }^{(10)}$.

Existe apenas um estudo clínico multicêntrico, randomizado e controlado por placebo com etanercepte em AIJ e seis estudos prospectivos não-controlados, com um total de 519 pacientes até 2006. Na fase aberta do estudo, $74 \%$ dos pacientes responderam ao etanercepte, preenchendo a resposta ACR Pediatric 30. Na segunda fase, duplo-cega, a reativação da AIJ foi estatisticamente maior no grupo placebo $(81 \%)$ versus grupo etanercepte (28\%) e a mediana do tempo de reativação da AIJ foi maior no grupo etanercepte (acima de 116 dias) versus placebo $\left(28\right.$ dias) ${ }^{(3)}$. Ao final de sete meses, incluindo fase aberta e fase duplo-cega, $80 \%$ dos pacientes preencheram ACR Pediatric 30, 72\% ACR Pediatric 50 e 44\% ACR Pediatric 70. Dos 58 pacientes com AIJ de evolução poliarticular ativa que participaram da fase de extensão aberta, 42 receberam etanercepte por um período de quatro anos e 26 por oito anos, com manutenção da resposta clínica ${ }^{(4)}$. A taxa de eventos adversos sérios foi de 0,13 por paciente/ano e a de infecções sérias foi 0,04 por paciente/ano, em um total de 225 pacientes/ano. Reações no local da injeção e infecções do trato respiratório superior foram os eventos adversos mais comumente relatados. Não houve síndrome lúpus-like, doença desmielinizante, malignidade ou óbito ${ }^{(11)}$. 


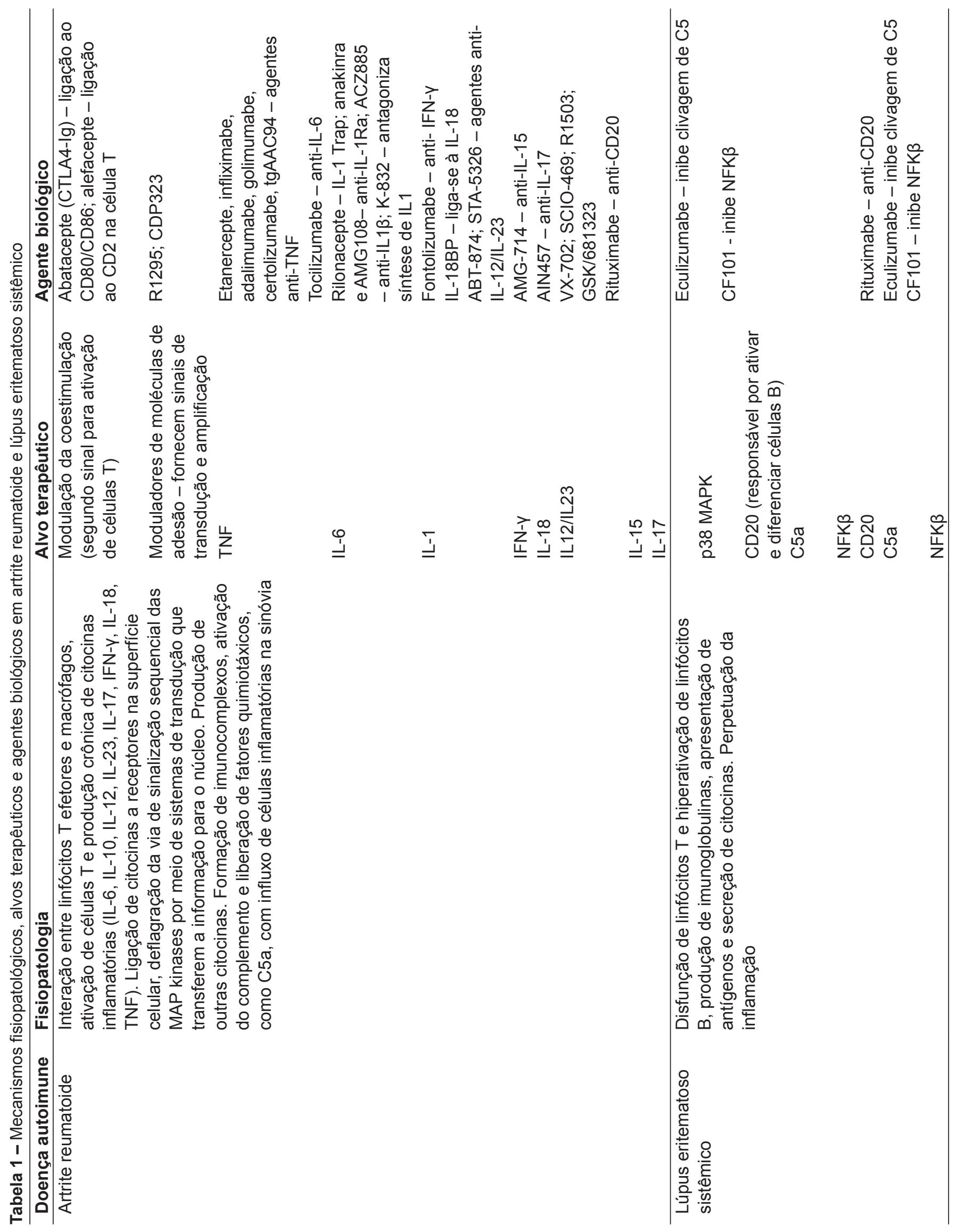


Tabela 2 - Agentes biológicos disponíveis para tratamento na artrite idiopática juvenil e suas características

\begin{tabular}{|c|c|c|c|c|c|c|c|}
\hline $\begin{array}{l}\text { Nome } \\
\text { genérico }\end{array}$ & $\begin{array}{l}\text { Nome } \\
\text { comercial }\end{array}$ & $\begin{array}{l}\text { Aprovação } \\
\text { para AlJ } \\
\text { (FDA) }\end{array}$ & $\begin{array}{l}\text { Aprovação } \\
\text { para AlJ } \\
\text { (Anvisa) }\end{array}$ & $\begin{array}{l}\text { Via de } \\
\text { administração e } \\
\text { dose }\end{array}$ & $\begin{array}{l}\text { Meia } \\
\text { vida }\end{array}$ & $\begin{array}{l}\text { Início } \\
\text { de ação }\end{array}$ & $\begin{array}{l}\text { Mecanismo } \\
\text { de ação }\end{array}$ \\
\hline Infliximabe & Remicade $^{\circledR}$ & Não & Não & $\begin{array}{l}\text { EV: } 3 \mathrm{mg} / \mathrm{kg} \mathrm{0,2,6} \\
\text { semanas e, após, a } \\
\text { cada } 8 \text { semanas }\end{array}$ & $\begin{array}{l}9,8 \\
\text { dias }\end{array}$ & $\begin{array}{l}7-14 \\
\text { dias }\end{array}$ & Anti-TNF \\
\hline Etanercepte & Enbre $^{\circledR}$ & 4-17 anos & Sim & SC: $0,4 \mathrm{mg} / \mathrm{kg}$ & $\begin{array}{l}4,8 \\
\text { dias }\end{array}$ & $\begin{array}{l}1-28 \\
\text { dias }\end{array}$ & Anti-TNF \\
\hline Adalimumabe & Humira $^{\circledR}$ & 4-17 anos & Não & SC: $24 \mathrm{mg} / \mathrm{m}^{2}$ & $\begin{array}{l}10-18 \\
\text { dias }\end{array}$ & $\begin{array}{l}1-14 \\
\text { dias }\end{array}$ & Anti-TNF \\
\hline Rituximabe & Mabthera ${ }^{\circledR}$ & Não & Não & NR & $N R$ & NR & $\begin{array}{l}\text { Depletor de } \\
\text { células B }\end{array}$ \\
\hline Tocilizumabe & NR & Não & Não & $\begin{array}{l}\mathrm{EV}: 2-8 \mathrm{mg} / \mathrm{kg} \text { a } \\
\text { cada } 2 \text { semanas }\end{array}$ & $\begin{array}{l}10,1 \\
\text { dias }\end{array}$ & NR & $\begin{array}{l}\text { Antagonista } \\
\text { do receptor de } \\
\text { IL-6 }\end{array}$ \\
\hline Abatacepte & Orencia $^{\circledR}$ & $6-17$ anos & Não & $\mathrm{EV}: 10 \mathrm{mg} / \mathrm{kg}$ & $\begin{array}{l}13,1 \\
\text { dias }\end{array}$ & NR & $\begin{array}{l}\text { Modulador } \\
\text { seletivo da } \\
\text { coestimulação }\end{array}$ \\
\hline
\end{tabular}

AIJ: artrite idiopática juvenil; EV: endovenoso; SC: subcutâneo; NR: não-relatado; FDA: Food and Drug Administration; Anvisa: Agência Nacional de Vigilância Sanitária.

Um estudo clínico multicêntrico, randomizado, duplocego, controlado por placebo, com infliximabe na dose inicial de $3 \mathrm{mg} / \mathrm{kg}$ associado a MTX, em 122 crianças com AIJ poliarticular persistente de quatro a 17 anos de idade mostrou que, apesar de um número maior de pacientes no grupo infliximabe ter atingido uma resposta ACR Pediatric 30 comparado ao grupo placebo $(63,8$ versus $49,2 \%)$ na fase aberta (duração de 14 semanas), não houve diferença estatística entre os dois grupos. Na $16^{a}$ semana, os pacientes do grupo placebo passaram a receber infliximabe $6 \mathrm{mg} / \mathrm{kg}$ e uma resposta ACR Pediatric 30 foi atingida por $73,2 \%$ deles. $\mathrm{Na} 52^{\mathrm{a}}$ semana, ACR Pediatric 50 e 70 foram observados em 69,6 e $51,8 \%$, respectivamente. O infliximabe foi geralmente bem tolerado. Entretanto, o perfil de segurança da dose de $3 \mathrm{mg} / \mathrm{kg}$ pareceu menos favorável do que a de $6 \mathrm{mg} /$ $\mathrm{kg}$, com frequência maior de eventos adversos sérios, reações infusionais (vômitos, febre, cefaleia, hipotensão), anticorpos anti-infliximabe, anticorpos antinucleares e anticorpos anti$\mathrm{DNA}^{(12)}$.

Outro estudo aberto, controlado, não-randomizado com 24 pacientes com AIJ poliarticular comparou etanercepte $(0,4 \mathrm{mg} / \mathrm{kg}$ ) com infliximabe (3 a $4 \mathrm{mg} / \mathrm{kg})$, associado a MTX e/ou a outras drogas modificadoras da doença: $78 \%$ dos pacientes atingiram o ACR Pedi 50 no grupo infliximabe e $89 \%$ no grupo etanercepte em 12 meses $^{(13)}$.

Em um estudo multicêntrico, randomizado, duplo-cego, controlado por placebo com adalimumabe, $74 \%$ dos pacien- tes (64 de 86 pacientes com AIJ poliarticular ativa, idades entre quatro a 17 anos) que não receberam MTX e 94\% dos que receberam MTX tiveram uma resposta ACR Pediatric 30 na $16^{a}$ semana e foram elegíveis para o tratamento duplocego. Entre os que receberam MTX, menos recidivas ocorreram no grupo adalimumabe (37\%) do que no grupo placebo (65\%). $\mathrm{Na} 48^{\mathrm{a}}$ semana, o percentual de pacientes tratados com MTX que obtiveram ACR Pediatric 30, 50, 70 ou 90 foi significantemente maior nos que receberam adalimumabe do que no grupo placebo. Eventos adversos sérios, possivelmente relacionados ao adalimumabe, ocorreram em 14 pacientes. Dentre esses, sete eram infecções sérias. Não houve óbitos, malignidades, infecções oportunísticas, casos de tuberculose, doenças desmielinizantes ou síndrome lúpus-like ${ }^{(14)}$.

Em um estudo multinacional, multicêntrico, randomizado, duplo-cego e controlado por placebo, 190 pacientes com AIJ receberam abatacepte $10 \mathrm{mg} / \mathrm{kg}$ via intravenosa numa fase aberta de quatro meses. Para pacientes recebendo MTX $(73,7 \%)$, as taxas de ACR Ped 30, 50, 70 e 90 foram $68,8,50,7,27,5$ e $12,3 \%$, respectivamente. Para pacientes sem MTX, as taxas foram 53,8, 46,2, 30,8 e 13,5\%, respectivamente. No período duplo-cego, 60 pacientes foram randomizados para receber abatacepte $10 \mathrm{mg} / \mathrm{kg}$ a cada 28 dias, durante seis meses ou até a recidiva da doença, e 62 foram randomizados para placebo. Recidivas ocorreram em $33 / 62(53 \%)$ pacientes que receberam placebo e em 12/60 (20\%) do grupo abatacepte. Os eventos adversos mais co- 
mumente relatados na fase aberta foram: cefaleia, náuseas, tosse, diarreia, infecção do trato respiratório superior e febre. Durante o período duplo-cego, a frequência de eventos adversos não diferiu entre os dois grupos. Eventos adversos foram relatados, respectivamente, em 37 (62\%) e 34 (55\%) pacientes do grupo abatacepte e placebo, sem diferença estatística. Apenas três eventos adversos sérios foram relatados no grupo controle. Não se observaram infecções sérias, infecções oportunísticas ou doenças autoimunes sérias ${ }^{(15)}$.

Em um estudo randomizado, duplo-cego, controlado por placebo, 56 crianças com AIJ de início sistêmico e idades entre dois e 19 anos receberam tocilizumabe $8 \mathrm{mg} /$ $\mathrm{kg}$ a cada duas semanas. As respostas ACR Pediatric 30, 50 e 70 foram atingidas por 51 (91\%), $48(86 \%)$ e 38 (68\%) pacientes, respectivamente. Apenas 4/23 (17\%) pacientes no grupo placebo mantiveram uma resposta ACR Pediatric 30 comparado a 16/20 (80\%) no grupo tocilizumabe. $\mathrm{Na} 48^{\mathrm{a}}$ semana, as taxas de ACR Pediatric 30, 50 e 70 foram atingidas por 47 (98\%), 45 (94\%) e 43 (90\%) de 48 pacientes, respectivamente. Eventos adversos frequentemente relatados foram sintomas de infecções do trato respiratório superior ou gastroenterite. Não houve óbitos, casos de síndrome de ativação macrofágica (uma das principais causas de óbitos em pacientes com esse subtipo de AIJ) ou tuberculose ${ }^{(16)}$.

Até o presente momento, não há estudos controlados com rituximabe para o tratamento da $\mathrm{AIJ}^{(8)}$.

$\mathrm{Na}$ Unidade de Reumatologia Pediátrica do Instituto da Criança do Hospital das Clínicas da Faculdade de Medicina da Universidade de São Paulo (USP), a experiência com uso de agentes biológicos tem focalizado o etanercepte em AIJ de curso poliarticular ativo. O seu emprego é recente, com uso crescente desde agosto de 2007. Essa droga tem sido bem tolerada por 24 pacientes e $90 \%$ deles preencheram o ACR Pediatric 30 com três meses de uso. Tanto o adalimumabe quanto o abatacepte foram aprovados pelo FDA, mas aguardam a aprovação pela Anvisa para uso futuro nos pacientes em nosso meio.

\section{Artrite relacionada à entesite}

A experiência com etanercepte em relação à artrite relacionada à entesite (ARE) resume-se a relatos de caso. Henrickson et al relataram oito pacientes com ARE refratária ao MTX ou à sulfassalazina, que receberam etanercepte por dois anos, com doses entre 0,2 e $0,8 \mathrm{mg} / \mathrm{kg}$, duas vezes por semana; quatro destes utilizaram MTX concomitantemente. Os sete pacientes que permaneceram no estudo apresentaram melho- ra mantida ao final de dois anos do número de articulações ativas, da anemia e da velocidade de hemossedimentação, com ausência de eventos adversos ${ }^{(17)}$.

\section{Miopatias inflamatórias idiopáticas}

Algumas crianças com dermatomiosite juvenil apresentam um curso de doença refratário a tratamento com múltiplas drogas. O TNF $\alpha$ foi encontrado em altos níveis em pacientes com dermatomiosite juvenil de longa evolução e com calcinoses. Riley $e t$ al ${ }^{(18)}$ descreveram cinco pacientes com doença refratária que receberam infliximabe via intravenosa na dose inicial de $3 \mathrm{mg} / \mathrm{kg}$ na semanas 0,2 e 6 e depois a cada oito semanas, sendo avaliados nas semanas 8 e 30 . Nos cinco pacientes, foi observada melhora da escala visual analógica (VAS), do Childhood Myositis Assessment Score (CMAS), do Childhood Health Assessment Questionnaire (CHAQ), do grau de movimentação articular e, em alguns casos, houve regressão das calcinoses e melhora das manifestações cutâneas.

Um estudo multicêntrico aberto e controlado com infliximabe associado a MTX foi realizado em pacientes com dermatomiosite juvenil e polimiosite juvenil de início recente e como tratamento inicial. Porém, o estudo foi terminado precocemente devido a uma elevada taxa de descontinuação por progressão da doença e ocorrência de reação infusional ${ }^{(19)}$.

$\mathrm{O}$ adalimumabe foi utilizado em dois pacientes (17 e 13 anos de idade) com miosite orbitária recorrente, refratária e não-responsiva ao tratamento imunossupressor habitual. Ambos melhoraram após introdução do adalimumabe, sendo possível a redução da dose do corticosteroide sem recidiva da doença ${ }^{(20)}$.

Uma análise retrospectiva de quatro pacientes pediátricos com idades entre dez e 17 anos com dermatomiosite juvenil tratados com rituximabe $\left(375 \mathrm{mg} / \mathrm{m}^{2}\right.$ semanalmente, via intravenosa, durante quatro semanas) mostrou que todos toleraram bem a medicação, com boa depleção das células B periféricas após as quatro semanas e sem infecções relacionadas ao tratamento. Três dos quatro pacientes apresentaram melhora clínica ${ }^{(21)}$.

O rituximabe foi também utilizado em três pacientes com dermatomiosite juvenil e manifestações cutâneas persistentes, apesar do uso prévio de imunossupressores, com melhora do quadro ${ }^{(22)}$.

\section{Lúpus eritematoso sistêmico juvenil}

$\mathrm{O}$ rituximabe tem sido proposto tanto para o tratamento das manifestações renais quanto das extrarrenais do LESJ. 
Estudo com 18 pacientes refratários a tratamentos convencionais mostrou melhora dos escores de atividade clínica, dos anticorpos anti-DNA, da função renal e da proteinúria em 93\% deles. Entretanto, cinco pacientes necessitaram de múltiplos cursos de rituximabe devido a recidivas ${ }^{(23)}$. Outro estudo com dez pacientes com LESJ na faixa etária pediátrica evidenciou infecções graves em três casos, um deles evoluiu para óbito ${ }^{(24)}$.

Em estudo recente, crianças e adolescentes com LESJ responderam bem à combinação de rituximabe e ciclofosfamida. Melhora significante ocorreu tanto na atividade da doença quanto nos parâmetros renais ${ }^{(5)}$.

Um protocolo semelhante, associando rituximabe e ciclofosfamida, foi aplicado a 12 pacientes com LESJ com idades entre 11 e 28 anos (média de 14 anos). Houve melhora no índice de atividade da doença, redução da prednisona, diminuição da VHS e aumento da fração C3. A terapia foi bem tolerada e não houve eventos adversos sérios ${ }^{(8)}$.

\section{Doença de Kawasaki}

Uma análise retrospectiva de pacientes com doença de Kawasaki, com persistência da febre e das manifestações clínicas após duas doses de gamaglobulina intravenosa e aspirina oral, mostrou desaparecimento da febre em 13/16 pacientes e redução dos níveis séricos de PCR em dez de 16 pacientes tratados com infliximabe. Não houve reações infusionais ou complicações associadas a este agente biológico ${ }^{(25)}$.

Um estudo multicêntrico randomizado comparou uma segunda infusão intravenosa de gamaglobulina $(2 \mathrm{~g} / \mathrm{kg})$ versus infliximabe $(5 \mathrm{mg} / \mathrm{kg})$ em 24 crianças (idades entre oito e 38 meses) com doença de Kawasaki aguda e febre persistente após tratamento inicial com gamaglobulina intravenosa. $\mathrm{O}$ desaparecimento da febre em 24 horas ocorreu em 11 de 12 pacientes tratados com infliximabe e oito de 12 retratados com gamaglobulina intravenosa. Não houve diferença significante entre os dois grupos com relação à eficácia e à segurança ${ }^{(26)}$.

\section{Arterite de Takayasu}

Quatro crianças com arterite de Takayasu (três refratárias ao tratamento convencional e uma como droga de primeira linha) foram tratadas com anti-TNF. Todas receberam infliximabe intravenoso como primeira droga anti-TNF na dose inicial de $5 \mathrm{mg} / \mathrm{kg}$, nas semanas 0,2 e 6 e, depois, a cada oito semanas, em associação com MTX ou azatioprina. Em dois desses pacientes, houve falha inicial, administrandose, então, o adalimumabe como segunda droga anti-TNF, via subcutânea na dose de $24 \mathrm{mg} / \mathrm{m}^{2}$ a cada duas semanas. No entanto, após um mês e meio e cinco meses de uso de adalimumabe, respectivamente, os pacientes apresentaram recidiva. Foi, assim, suspenso o adalimumabe e reintroduzido o infliximabe, entrando os dois pacientes em remissão. Nos outros dois pacientes que receberam exclusivamente infliximabe como anti-TNF, a resposta foi parcial ${ }^{(27)}$.

\section{Uveítes crônicas}

Outra indicação para o uso de biológicos é a uveíte anterior crônica ou iridociclite crônica. Essa é uma complicação comum em crianças do sexo feminino com AIJ oligoarticular em idade pré-escolar, e com presença de anticorpos antinucleares. Entretanto, a uveíte/iridiciclite pode ser idiopática ou associada a outras doenças reumatológicas.

O infliximabe é geralmente administrado na dose de 3 a $10 \mathrm{mg} / \mathrm{kg}$, porém doses de 10 a $20 \mathrm{mg} / \mathrm{kg}$ foram utilizadas com sucesso e poucos eventos adversos num grupo de 17 crianças com uveíte crônica ${ }^{(28)}$.

O adalimumabe foi aplicado a 18 crianças com uveíte anterior crônica, sendo 17 com AIJ e um sem doença associada. O tratamento foi eficaz em 16 pacientes ${ }^{(29)}$. Dois estudos com adalimumabe para tratar uveíte na infância (a maioria associada à AIJ), em um total de 33 pacientes, mostraram atenuação da inflamação em 80 a $88 \%$, sendo possível reduzir ou suspender os corticosteroides e/ou imunossupressores associados $^{(28,29)}$.

Tugal-Tuktun et al ${ }^{(30)}$ relataram 20 crianças com uveíte não-infecciosa tratadas com infliximabe como terapia imunomoduladora inicial (quatro pacientes) ou após uso de imunossupressores (16 crianças). A inflamação ocular foi controlada em curto prazo em todas as crianças, porém ocorreu resistência ao tratamento em quatro delas em longo prazo.

$\mathrm{Na}$ fase inicial das uveítes, os três anti-TNF disponíveis parecem ser eficazes, entretanto, nos casos graves e crônicos os anticorpos (infliximabe e adalimumabe) se mostraram mais eficazes do que o receptor solúvel (etanercepte).

\section{Doença inflamatória intestinal}

A maioria dos estudos em crianças e adolescentes com doença de Crohn tem utilizado infliximabe (no esquema de infusão inicial de zero, duas e seis semanas e, depois, a cada 
oito semanas) como terapia preferencial nos casos não-responsivos aos corticosteroides, 6-mercaptopurina, azatioprina e/ou MTX. A resposta clínica foi atingida em 52 a 100\% de pacientes com doença luminal e os corticosteroides foram suspensos em quase $80 \%$ deles entre 24 e 30 semanas, após $\mathrm{o}$ início das infusões com infliximabe ${ }^{(31)}$. Kugathasan et a ${ }^{(32)}$ avaliaram prospectivamente pacientes com doença de Crohn precoce, que apresentaram uma resposta mais prolongada ao infliximabe comparados àqueles com doença tardia.

Recentemente, o infliximabe tem sido administrado para crianças com retocolite ulcerativa idiopática ${ }^{(33)}$, entretanto, o número de pacientes pediátricos tratados com agentes biológicos relatados até o presente momento é menor do que aqueles com doença de Crohn. O infliximabe induz uma melhora a curto prazo em quase dois terços das crianças com retocolite ulcerativa idiopática crônica ou fulminante. Uma resposta clínica mantida foi relatada em quase 63 a $75 \%$ dos pacientes, a maioria necessitando de retratamento com infliximabe ${ }^{(34)}$.

Pesquisa recente avaliou a segurança de infliximabe em 111 crianças e adolescentes com doença inflamatória intestinal que receberam 594 infusões do medicamento. A ocorrência de reações infusionais foi observada em $1,5 \%$ das administrações ${ }^{(35)}$.

Um estudo retrospectivo avaliou a resposta a adalimumabe em dez pacientes com doença inflamatória intestinal (três com retocolite ulcerativa idiopática e sete com doença de Crohn). Destes, oito haviam respondido inicialmente ao infliximabe, sendo a mediana de tempo entre o início de infliximabe e o de adalimumabe de 24 meses. Oito pacientes responderam ao adalimumabe ( 40 ou $80 \mathrm{mg}$ a cada duas semanas). As médias do Pediatric Crohn's Disease Activity Index (PCDAI) nos pacientes com doença de Crohn antes e após o adalimumabe foram, respectivamente, 12 e 4,2. As médias do Lichtiger Colitis Activity Index (LCAI) nos pacientes com retocolite ulcerativa idiopática antes e após o adalimumabe foram, respectivamente, 9 e 5,1. Os corticosteroides foram suspensos em quatro dos sete pacientes, um a dez meses após o início do adalimumabe. Um paciente com doença de Crohn e outro paciente com retocolite ulcerativa idiopática não responderam ao adalimumabe, necessitaram de cirurgia e permaneceram em uso de corticosteroides ${ }^{(36)}$.

\section{Linfoma não Hodgkin}

Vários estudos têm demonstrado a eficácia do rituximabe intravenoso em pacientes com malignidades linfoides originadas de células B, incluindo leucemia linfocítica crô- nica e formas agressivas (como o linfoma difuso de grandes células B) e indolentes (por exemplo, linfoma folicular) de $\mathrm{LNH}^{(37,38)}$.

No entanto, a droga ainda não foi aprovada para uso em leucemia linfocítica crônica. O rituximabe está indicado para o tratamento de LNH CD20+ de baixo grau ou folicular como monoterapia em pacientes adultos refratários ou com recidivas ou em associação com o esquema CVP (ciclofosfamida, vincristina e prednisona) naqueles previamente nãotratados e como terapia de manutenção em pacientes com doença refratária ou recidivante. É também indicado como primeira linha para tratamento de LNH difuso de grandes células B em combinação com o esquema CHOP (ciclofosfamida, doxorubicina, vincristina e prednisona $)^{(37,38)}$.

O seu uso em crianças e adolescentes é considerado offlabel. Em estudos de crianças em quimioterapia isolada sem rituximabe, taxas de sobrevida de mais de $90 \%$ foram atingidas naquelas com linfoma difuso de grandes células B. Contudo, a adição de rituximabe teve um efeito benéfico, semelhante à adição de etoposide ao esquema $\mathrm{CHOP}$, de forma que, talvez, o rituximabe em crianças possa substituir, nesse caso, agentes quimioterápicos que induzem sérios efeitos adversos, agudos ou crônicos ${ }^{(37,38)}$.

\section{PTI crônica}

O rituximabe mostrou eficácia mantida em 11/36 crianças e adolescentes com PTI crônica grave e refratária ao tratamento convencional, seguidos por um ano, havendo manutenção da contagem de plaquetas acima de $50.000 / \mathrm{mm}^{3}$. Oito de 11 pacientes inicialmente responsivos mantiveram uma contagem de plaquetas acima de $150.000 / \mathrm{mm}^{(3,39)}$.

\section{Segurança}

As contraindicações absolutas e relativas dos agentes biológicos em crianças são as mesmas dos adultos. Dentre elas, destacam-se: presença de infecção ativa aguda ou crônica (especialmente tuberculose), insuficiência cardíaca grau III/IV, doenças desmielinizantes, gravidez, história recente de neoplasia ( $<5$ anos) e neurite óptica. Vacinas vivas não devem ser administradas durante o tratamento com agentes biológicos e, de preferência, utilizadas até três meses antes da administração da droga ${ }^{(40,41)}$.

O uso concomitante de dois ou mais agentes biológicos deve ser bem avaliado individualmente devido ao risco aumentado de eventos adversos, particularmente infecções ${ }^{(40,41)}$. 
Entretanto, o uso de pelo menos dois biológicos pode eventualmente ser empregado, dependendo da gravidade e da refratariedade do quadro de base.

Outra preocupação se refere ao risco de reativação de infecção latente por Mycobacterium tuberculosis, principalmente com o uso de terapia anti-TNF. Antes da introdução de um agente biológico deve-se, obrigatoriamente, avaliar epidemiologia para tuberculose, solicitar teste de Mantoux e radiografia de tórax ${ }^{(40,41)}$. Além disso, diante de qualquer infecção aguda/crônica ou necessidade de cirurgia, o início da terapia com agente biológico deve ser adiado ou suspenso temporariamente ${ }^{(40,41)}$.

Em caso de gestação, os agentes biológicos devem ser imediatamente suspensos. Estudos futuros serão necessários para determinar a sua segurança na gravidez. A contracepção precisa ser sempre orientada na paciente adolescente com doença crônica e que utilize agente biológico ${ }^{(42)}$.

\section{Referências bibliográficas}

1. Voulgari PV. Emerging drugs for rheumatoid arthritis. Expert Opin Emerg Drugs 2008;13:175-96.

2. Efthimiou P, Markenson JA. Role of biological agents in immune-mediated inflammatory diseases. South Med J 2005;98:192-204.

3. Mok CC. Emerging drug therapies for systemic lupus erythematosus. Expert Opin Emerg Drugs 2006;11:597-608.

4. Olsen NJ, Stein CM. New drugs for rheumatoid arthritis. N Engl J Med 2004;350:2167-79.

5. Macdermott EJ, Adams A, Lehman TJ. Systemic lupus erythematosus in children: current and emerging therapies. Lupus 2007;16:677-83.

6. Marks SD, Patey S, Brogan PA, Hasson N, Pilkington C, Woo P et al. B lymphocyte depletion therapy in children with refractory systemic lupus erythematosus. Arthritis Rheum 2005;52:3168-74.

7. Barillas-Arias L, Adams A, Angeles S, MacDermott EJ, Barinstein L, Lehman TJ. Combination of rituximab and cyclophosphamide for the treatment of childhood onset systemic lupus erythematosus (Abstract). Arthritis Rheum 2006;54:S689-90.

8. Gartlehner G, Hansen RA, Jonas BL, Thieda P, Lohr KN. Biologics for the treatment of juvenile idiopathic arthritis: a systematic review and critical analysis of the evidence. Clin Rheumatol 2008;27:67-76.

9. Martin P, Medeiros AC, Goldenstein-Schainberg C. The role of tumor necrosis factor inhibitors in the treatment of juvenile idiopathic arthritis. Rev Bras Reumatol 2006;46:126-33.

10. Ruperto N, Martini A. Network in pediatric rheumatology: the example of Pediatric Rheumatology International Trials Organization. Georgian Med News 2008:68-74.

11. Lovell DJ, Reiff A, llowite NT, Wallace CA, Chon Y, Lin SL et al. Safety and efficacy of up to eight years of continuous etanercept therapy in patients with juvenile rheumatoid arthritis. Arthritis Rheum 2008;58:1496-504.

12. Ruperto N, Lovell DJ, Cuttica R, Wilkinson N, Woo P, Espada G et al. A randomized, placebo-controlled trial of infliximab plus methotrexate for the treatment of polyarticular-course juvenile rheumatoid arthritis. Arthritis Rheum 2007; 56:3096-106.

13. Lahdenne $P$, Vähäsalo, $P$, Honkanen V. Infliximab or etanercept in the treatment of children with refractory juvenile idiopathic arthritis: an open label study. Ann Rheum Dis 2003;62:245-7.

\section{Conclusões}

Os agentes biológicos têm demonstrado eficácia no tratamento de doenças autoimunes pediátricas, como AIJ, miopatias idiopáticas inflamatórias, lúpus eritematoso juvenil, vasculites, uveítes crônicas, DII, e PTI crônica, assim como no LNH.

A terapia com essas drogas não é isenta de riscos, apresenta custo elevado e deve ser individualizada e utilizada de forma criteriosa, tanto com seu uso isolado como combinado com outros medicamentos (como anti-inflamatórios não-hormonais, corticosteroides, drogas modificadoras do curso da doença/imunossupressores e gamaglobulina endovenosa, entre outros), para o controle adequado das doenças refratárias.

O especialista pediátrico deve ser preferencialmente o responsável pela indicação e seguimento dos pacientes e deve discutir eficácia e potenciais eventos adversos em curto, médio e longo prazo.

14. Lovell DJ, Ruperto N, Goodman S, Reiff A, Jung L, Jarosova K et al. Adalimumab with or without methotrexate in juvenile rheumatoid arthritis. $\mathrm{N}$ Engl J Med 2008;359:810-20.

15. Ruperto N, Lovell DJ, Quartier P, Paz E, Rubio-Pérez N, Silva CA et al. Abatacept in children with juvenile idiopathic arthritis: a randomized, double-blind, placebo-controlled withdrawal trial. Lancet 2008;372: 383-91.

16. Yokota S, Imagawa T, Mori M, Miyamae T, Aihara Y, Takei S et al. Efficacy and safety of tocilizumab in patients with systemic-onset juvenile idiopathic arthritis: a randomised, double-blind, placebo-controlled, withdrawal phase III trial. Lancet 2008;371:998-1006.

17. Henrickson M, Reiff A. Prolonged efficacy of etanercept in refractory enthesitisrelated arthritis. J Rheumatol 2004;31:2055-61.

18. Riley P, McCann LJ, Maillard SM, Woo P, Murray KJ, Pilkington CA. Effectiveness of infliximab in the treatment of refractory juvenile dermatomyositis with calcinosis. Rheumatology (Oxford) 2008;47:877-80.

19. Hengstman GJ, De Bleecker JL, Feist E, Vissing J, Denton CP, Manoussakis $\mathrm{MN}$ et al. Open-label trial of anti-TNF-alpha in dermato- and polymyositis treated concomitantly with methotrexate. Eur Neurol 2008;59:159-63.

20. Adams AB, Kazim M, Lehman TJ. Treatment of orbital myositis with adalimumab (Humira). J Rheumatol 2005;32:1374-5.

21. Cooper MA, Willingham DL, Brown DE, French AR, Shih FF, White AJ. Rituximab for the treatment of juvenile dermatomyositis: a report of four pediatric patients. Arthritis Rheum 2007;56:3107-11.

22. Dinh HV, McCormack C, Hall S, Prince HM. Rituximab for the treatment of the skin manifestations of dermatomyositis: a report of 3 cases. J Am Acad Dermatol 2007;56:148-53.

23. Nwobi O, Abitbol CL, Chandar J, Seeherunvong W, Zilleruelo G. Rituximab therapy for juvenile-onset systemic lupus erythematosus. Pediatr Nephrol 2008;23:413-9.

24. El-Hallak M, Binstadt BA, Leichtner AM, Bennett CM, Neufeld EJ, Fuhlbrigge $\mathrm{RC}$ et al. Clinical effects and safety of rituximab for treatment of refractory pediatric autoimmune diseases. J Pediatr 2007;150:376-82.

25. Burns JC, Mason WH, Hauger SB, Janai H, Bastian JF, Wohrley JD et al. Infliximab treatment for refractory Kawasaki syndrome. J Pediatr 2005;146:662-7. 
26. Burns JC, Best BM, Mejias A, Mahony L, Fixler DE, Jafri HS et al. Infliximab Treatment of Intravenous Immunoglobulin-Resistant Kawasaki Disease. J Pediatr 2008;153:833-8.

27. Filocamo G, Buoncompagni A, Viola S, Loy A, Malattia C, Ravelli A et al. Treatment of Takayasu's arteritis with tumor necrosis factor antagonists. J Pediatr 2008;153:432-4.

28. Imrie FR, Dick AD. Biologics in the treatment of uveitis. Curr Opin Ophthalmol 2007;18:481-6.

29. Biester S, Deuter C, Michels H, Haefner R, Kuemmerle-Deschner J, Doycheva $\mathrm{D}$ et al. Adalimumab in the therapy of uveitis in childhood. Br J Ophthalmol 2007;91:319-24.

30. Tugal-Tutkun I, Ayranci O, Kasapcopur O, Kir N. Retrospective analysis of children with uveitis treated with infliximab. J AAPOS 2008;12:611-3.

31. Baldassano R, Braegger CP, Escher JC, DeWoody K, Hendricks DF, Keenan GF et al. Infliximab (REMICADE) therapy in the treatment of pediatric Crohn's disease. Am J Gastroenterol 2003;98:833-8.

32. Kugathasan S, Werlin SL, Martinez A, Rivera MT, Heikenen JB, Binion DG. Prolonged duration of response to infliximab in early but not late pediatric Crohn's disease. Am J Gastroenterol 2000;95:3189-94.

33. Russell GH, Katz AJ. Infliximab is effective in acute but not chronic childhood ulcerative colitis. J Pediatr Gastroenterol Nutr 2004;39:166-70.

34. Schwarzer A, Ricciardelli I, Kirkham S, Binnie K, Shah N, Elawad MA et al. Management of fulminating ulcerative colitis in childhood with chimeric antiCD25 antibody. J Pediatr Gastroenterol Nutr 2006;42:245-8.
35. Jacobstein DA, Markowitz JE, Kirschner BS, Ferry G, Cohen SA, Gold BD et al. Premedication and infusion reactions with infliximab: results from a pediatric inflammatory bowel disease consortium. Inflamm Bowel Dis 2005;11: 442-6.

36. Noe JD, Pfefferkorn M. Short-term response to adalimumab in childhood inflammatory bowel disease. Inflamm Bowel Dis 2008;14:1683-7.

37. Plosker GL, Figgitt DP. Rituximab: a review of its use in non-Hodgkin's lymphoma and chronic lymphocytic leukaemia. Drugs 2003;63:803-43.

38. Reiter A, Klapper W. Recent advances in the understanding and management of diffuse large B-cell lymphoma in children. $\mathrm{Br} \mathrm{J}$ Haematol 2008;142: 329-47.

39. Mueller BU, Bennett CM, Feldman HA, Bussel JB, Abshire TC, Moore TB et al. One year follow-up of children and adolescents with chronic immune thrombocytopenic purpura (ITP) treated with rituximab. Pediatr Blood Cancer 2009;52:259-62.

40. Santos MJ, Fonseca JE, Canhão H, Conde M, Vieira MJ, Costa L et al. Consensos para início e manutenção de terapêutica biológica na artrite idiopática juvenil. Acta Reum Port 2007;32:43-7.

41. Bértolo MB, Brenol CV, Goldenstein-Schainberg C, Neubarth F, Lima $\mathrm{FA}$, Laurindo IM et al. Atualização do consenso brasileiro no diagnóstico e tratamento da artrite reumatóide. Rev Bras Reumatol 2007;47:1 51-9.

42. Tandon VR, Sharma S, Mahajan A, Khajuria V, Kumar A. Pregnancy and rheumatoid arthritis. Indian J Med Sci 2006;60:334-44. 